\title{
Clinical implications of serum Mac-2-binding protein glycan isomer as a novel biomarker of advanced hepatic fibrosis in diabetes
}

\author{
Huiyul Park ${ }^{1} \wedge$, Dae Won Jun ${ }^{2}$, Hoon-Ki Park ${ }^{1 \wedge}$, Mimi Kim $^{3} \wedge$ \\ ${ }^{1}$ Department of Family Medicine, Hanyang University College of Medicine, Seoul, Korea; ${ }^{2}$ Department of Internal Medicine, Hanyang University \\ College of Medicine, Seoul, Korea; ${ }^{3}$ Department of Radiology, Hanyang University College of Medicine, Seoul, Korea \\ Contributions: (I) Conception and design: DW Jun; (II) Administrative support: DW Jun; (III) Provision of study materials or patients: HK Park; (IV) \\ Collection and assembly of data: H Park; (V) Data analysis and interpretation: H Park, M Kim; (VI) Manuscript writing: All authors; (VII) Final \\ approval of manuscript: All authors. \\ Correspondence to: Dr. Dae Won Jun, Department of Internal Medicine, Hanyang University College of Medicine, 222 Wangsimni-ro, Seongdong-gu, \\ Seoul, 133-791, Korea. Email: noshin@hanyang.ac.kr.
}

Background: Appropriate strategy for screening, identification, and linkage to care of patients with advanced fibrosis in the general population is a current issue. The aim of this study was to find reference values and the clinical role of Mac-2 binding protein glycan isomer (M2BPGi) in a health check-up setting.

Methods: This study was designed as cross-sectional study. Adult subjects $(n=1,073)$ who underwent a health check-up were included in the final analysis, and 952 subjects with risk factors for liver disease and insufficient data were excluded. M2BPGi quantification was based on a lectin antibody sandwich immunoassay. Fatty liver was diagnosed using abdominal sonography.

Results: The reference value of M2BPGi was $0.5-1.0$ cut off index (COI) in the average risk group. Serum M2BPGi showed a positive correlation with metabolic parameters as well as age. Prevalence of abnormal M2BPGi (>1.0) was higher in low muscle mass (4.7\%, vs. $17.4 \%, \mathrm{P}=0.002)$, metabolic syndrome (14.2\% vs. $30.4 \%, \mathrm{P}=0.003)$, and hypertension $(21.8 \%$, vs. $58.7 \%, \mathrm{P}<0.001)$ compared to healthy controls. M2BPGi was positively correlated with estimated fibrosis values such as FIB-4 $(\mathrm{R}=0.293, \mathrm{P}<0.001)$ and NAFLD fibrosis score $(\mathrm{R}=0.248, \mathrm{P}<0.001)$. Although the prevalence of advanced fibrosis in the total population was just $1.6 \%$ (FIB-4 >2.65), the prevalence of advanced fibrosis increased to 50\% in the high M2BPGi (>1.0) group with diabetes. This value was 31.25 times higher than in the total population group.

Conclusions: The results indicated a high possibility of advanced hepatic fibrosis in diabetic subjects with abnormal M2BPGi level (>1.0).

Keywords: Non-alcoholic fatty liver disease (NAFLD); diabetes mellitus; type 2; mac-2 binding protein glycan isomer; liver fibrosis

Submitted Jul 09, 2020. Accepted for publication Sep 30, 2020.

doi: $10.21037 /$ atm-20-5216

View this article at: http://dx.doi.org/10.21037/atm-20-5216

\footnotetext{
^ ORCID: Huiyul Park, 0000-0001-5044-8688; Dae Won Jun, 0000-0002-2875-6139; Hoon-Ki Park, 0000-0002-8242-0943; Mimi Kim, 0000-0002-8266-0226.
} 


\section{Introduction}

Non-alcoholic fatty liver disease (NAFLD) is perceived as a major cause of chronic liver disease worldwide $(1,2)$. Due to progression of illness, it is important to identify advanced liver fibrosis in the general population. Liver biopsy is a gold standard for assessing fibrosis stage, but it has several limitations, such as invasiveness, sampling error, and associated cost.

To date, various non-invasive approaches have been developed and include transient elastography such as the FibroScan (3) and magnetic resonance elastography (4). Various scoring systems such as the AST-to-platelet ratio index (APRI) (5), FIB-4 index (6), and NAFLD Fibrosis Score (7) and serologic markers including cytokeratin-18 (3), ASAGP (8), and Mac-2 binding protein have been studied to predict advanced fibrosis subjects in the general population. However, to date, no single test or algorithm has been accepted widely for use in the general population.

Glycoproteins are synthesized, secreted, and metabolized in the liver, and increased levels are prominent in liver disease $(9,10)$. Among serum glycoproteins for liver fibrosis, Mac2 binding protein glycosylation isomer (M2BPGi, Wisteria floribunda agglutinin-positive Mac-2 binding protein) has been recently studied as a useful serum marker for evaluating liver fibrosis in various chronic liver diseases such as chronic hepatitis $\mathrm{C}$ virus infection, autoimmune hepatitis, primary biliary cholangitis, and NAFLD (9-11). In a biopsy-proven cohort, M2BPGi differentiated patients with NASH at a cutoff 0.95 (AUROC 0.759) and determined significant fibrosis $(>\mathrm{F} 2)$ at a cut-off 1.0 (AUROC 0.758 ) from patients with NAFLD (11).

However, most studies have been focused on the role of M2BPGi for diagnosing NASH or predicting advanced fibrosis in patients in high risk groups. Therefore, it is important to develop screening strategies for patients with advanced liver fibrosis in the general population. A few studies have evaluated the clinical characteristics of $\mathrm{M} 2 \mathrm{BPGi}$ in average risk groups. Moreover, cut-off value of M2BPGi for predicting advanced fibrosis can differ according to liver fibrosis aetiology. There is currently limited research on whether M2BPGi can be applied for screening advanced liver fibrosis patients in the general population. The aim of this study was to determine the clinical role of M2BPGi in a health check-up clinic setting. We present the following article in accordance with the STROBE reporting checklist (available at http://dx.doi.org/10.21037/atm-20-5216).

\section{Methods}

\section{Study design}

This study was designed as cross-sectional study. The medical records of health check-up examiner at Hanyang University Hospital were collected for analysis, retrospectively. Only serum M2BPGi was measured additionally by using remnant blood samples. The study was conducted in accordance with the Declaration of Helsinki (as revised in 2013). The Institutional Review Board (IRB) of Hanyang University Medical Centre approved this study protocol (IRB No. 2018-12-020). The protocol was also registered at the Clinical Research Information Service (https://cris.nih.go.kr/cris, Registration No. KCT0004462). The informed consent was waived, because suspected harm to participants was minimal or none.

\section{Inclusion and exclusion criteria}

In this study, a total of 2,025 of adult subjects who underwent a health check-up at International Healthcare Service at Hanyang University Medical Centre between March 2019 and June 2019 were initially recruited into the study. We collected the data and excluded foreigners $(\mathrm{n}=339)$, subjects with insufficient remnant blood samples $(n=53)$, subjects with missing data for physical measurement including skeletal muscle mass $(n=20)$, subjects with abdominal ultrasound $(n=48)$, and those with alcohol consumption ( $n=28)$ or serum M2BPGi level $(n=270)$. We also excluded the high-risk liver disease population, subjects that had positive serologic markers of hepatitis B or hepatitis $C$ viruses, those that were self-reported HBV carriers $(n=65)$ or subjects whose weekly alcohol consumption was greater than $210 \mathrm{~g}$ for men or greater than $140 \mathrm{~g}$ for women $(\mathrm{n}=160)$. Finally, 1,073 subjects were included in this study (Figure 1).

\section{Clinical variables and laboratory evaluations}

Personal medical and medication history, smoking history, exercise, and alcohol consumption were collected through self-reported surveys. Body weight and height were measured, and body mass index (BMI) was calculated as weight in kilograms $(\mathrm{kg})$ divided by height in metres squared $\left(\mathrm{m}^{2}\right)$. Waist circumference (WC) was measured at the narrowest point between the iliac crest and the lower rib margin. Blood pressure was measured at rest in 


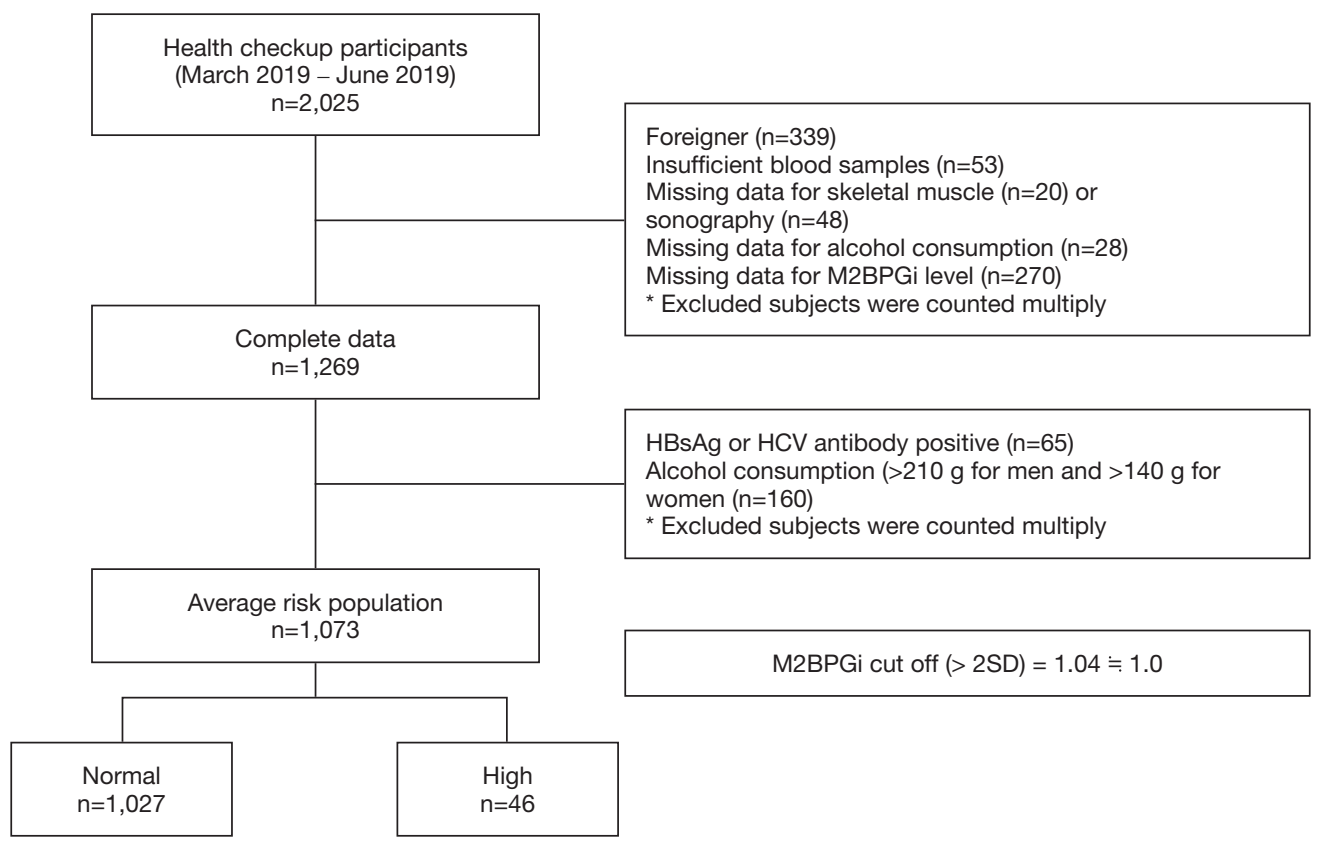

Figure 1 Study flow diagram. M2BPGi, Mac-2 binding protein glycosylation isomer.

a sitting position. Body composition was analysed using Bioelectrical Impedance Analysis (BIA; InBody 720 body composition analysis). The skeletal muscle index (SMI) was calculated by dividing total appendicular skeletal muscle (ASM), which is the sum of skeletal muscle in the bilateral upper and lower four limbs $(\mathrm{kg})$, by square of height (= total ASM/height ${ }^{2}$ ). The cut-off values for low muscle mass were defined by hSMI $\left(<6.58 \mathrm{~kg} / \mathrm{m}^{2}\right.$ for men and $<4.56 \mathrm{~kg} / \mathrm{m}^{2}$ for women) (12). In addition, the cut-off values for low muscle mass were defined by wSMI $(<29.1 \%$ for men and $<23.0 \%$ for women) (12).

\section{Estimated hepatic fat and fibrosis formulae}

Fatty Liver Index (FLI) was calculated with descriptive statistical analysis using this equation: $\left\{\mathrm{e}^{\mathrm{y}} /\left(1+\mathrm{e}^{\mathrm{y}}\right) \times 100\right.$, where $\mathrm{y}=0.953 \times \ln [\mathrm{TG}(\mathrm{mg} / \mathrm{dL})]+0.139 \times \mathrm{BMI}\left[\mathrm{kg} / \mathrm{m}^{2}\right]+0.718$ $\times \ln [\mathrm{GGT}(\mathrm{U} / \mathrm{L})]+0.053 \times \mathrm{WC}(\mathrm{cm})-15.745\}(13)$. The fibrosis-4 (FIB-4) index was calculated using the following equation: [age $\times$ AST $(\mathrm{U} / \mathrm{L}) /$ platelet count $\left(\times 10^{9} / \mathrm{L}\right) / \mathrm{ALT}$ (U/L)] (6). FIB-4 grades were divided into 0,1 , and 2 using cut-off values of 1.3 and 2.67. The NAFLD fibrosis score (NFS) was calculated using this equation: $[-1.675+0.037 \times 3$ age (years) $+0.094 \times$ BMI $\left(\mathrm{kg} / \mathrm{m}^{2}\right)+1.13 \times$ impaired fasting glycemia/diabetes mellitus (yes $=1$, no $=0)+0.99 \times$ AST/ALT $-0.013 \times$ platelet count $\left.\left(\times 10^{9} / \mathrm{L}\right)-0.66 \times \mathrm{Alb}(\mathrm{g} / \mathrm{dL})\right](7)$. NFS grades are divided into 0,1 , and 2 using cut-off values of -1.455 and 0.675 . Grades 0,1 , and 2 of the FIB-4 index and NFS indicate that advanced fibrosis can be excluded, further examination is needed, and advanced fibrosis, respectively. Impaired fasting glucose (IFG) was defined as FBS of $100-125 \mathrm{mg} / \mathrm{dL}$. The presence of type 2 diabetes mellitus (T2DM) was defined as FBS $126 \mathrm{mg} / \mathrm{dL}, \mathrm{HbA1c} \geq 6.5 \%$, or treatment with anti-diabetic drugs. AST to Platelet ratio index (APRI) was calculated using the following equation: (AST in $\mathrm{IU} / \mathrm{L}) /\left(\right.$ AST upper limit of normal in IU/L)/(Platelets in $10^{9} / \mathrm{L}$ ). $40 \mathrm{IU} / \mathrm{L}$ was used as upper limit of normal. APRI grades are divided into 0,1 , and 2 using cut-off values of 0.7 and 1.0. APRI grade 1 and 2 indicate significant and severe liver fibrosis, respectively (14).

\section{M2BP measurement}

The M2BPGi quantification was based on a lectin antibody sandwich immunoassay performed using a fully automatic immune-analyser (HISCL-2000i; Sysmex Co., Hyogo, Japan). The measured values of Wisteria floribunda agglutinin-positive $\left(\mathrm{WFA}^{+}\right)-\mathrm{M} 2 \mathrm{BP}$ conjugated to WFA were indexed with the obtained values using the following equation: Cut-off index $(\mathrm{COI})=\left[\left(\mathrm{WFA}^{+}-\mathrm{M} 2 \mathrm{BP}\right)_{\text {sample }^{-}}\right.$ $\left.\left(\mathrm{WFA}^{+}-\mathrm{M} 2 \mathrm{BP}\right)\right]_{\mathrm{NC}} /\left[\left(\mathrm{WFA}^{+}-\mathrm{M} 2 \mathrm{BP}\right)_{\mathrm{PC}^{-}}\left(\mathrm{WFA}^{+}-\mathrm{M} 2 \mathrm{BP}\right)_{\mathrm{NC}}\right]$, where $\left(\mathrm{WFA}^{+}-\mathrm{M} 2 \mathrm{BP}\right)_{\text {sample }}$ was the $\mathrm{WFA}^{+}-\mathrm{M} 2 \mathrm{BP}$ count for 
the serum sample, $\mathrm{PC}$ was the positive control, and $\mathrm{NC}$ was the negative control. The $\mathrm{PC}$ was supplied as a calibration solution preliminarily standardized to yield a COI value of 1.0.

\section{Metabolic syndrome and NAFLD definitions}

In this study, the criteria from the National Cholesterol Education Program's Adult Treatment Panel III (NCEP-ATP III) were followed, with the exception of abdominal obesity, which was based on waist circumference, for diagnosing metabolic syndrome (15). The waist circumference values used were $\geq 85 \mathrm{~cm}$ for women and $\geq 90 \mathrm{~cm}$ for men, which are the standard measurements for the Korean population (16). The presence of hypertension was defined as systolic/ diastolic blood pressure $\geq 140 / 90 \mathrm{mmHg}$, or treatment with anti-hypertensive drugs.

NAFLD cases were diagnosed by abdominal ultrasonography among subjects without excessive alcohol consumption or viral or genetic liver disease. The degree of fatty liver was graded as normal, mild, moderate or, severe based on the degree of fat infiltration (17).

\section{Statistical analysis}

Participants were divided into two groups (Abnormal M2BPGi/Normal groups) using a cut-off value of 1.0 ( $>2 \mathrm{SD}$ ). Continuous and categorical variables are presented as mean (SD) and number (percent), respectively. For comparing the mean values between the groups, a $t$-test or ANOVA were used for continuous variables. Categorical variables were compared using chi-square tests. Fisher's Exact test was used for categorical variables for which $20 \%$ or more of the cells had an expected frequency less than five. Pearson correlation test was used to calculate correlation coefficient between serum M2BPGi and liver fibrosis formulae. Especially, odds ratio $(\mathrm{OR})$ with $95 \%$ confidence interval $(\mathrm{CI})$ for risk factor analysis of high M2BPGi were calculated using chi-square tests or Fisher's Exact test. For all analyses, P value less than 0.05 were considered statistically significant. Statistical analyses were performed using SPSS version 26 for Windows (SPSS Inc., Chicago, IL, USA). All subjects who have any missing data were excluded in analysis.

\section{Results}

\section{Characteristics of the study population}

One thousand seventy-three subjects were included in the analysis, and 952 subjects with risk factors for liver disease and insufficient data were excluded (Figure 1). Because the aim of this study to know how well serum M2BPGi represents hepatic fibrosis, the baseline characteristics, demographic data and main outcomes such as FIB-4, NFS, AST or APRI of the 1,073 study subjects according to M2BPGi level, and Pearson correlations between M2BPGi and other variables are presented in Table 1 . The mean age of the subjects was 47.11 years, and the proportion of males was $58.3 \%$. The mean value and standard deviation of serum M2BPGi were 0.511 and 0.264 , respectively. The upper normal limit (>2SD) was 1.04 (Figure $2 A, B$ ).

\section{Clinical characteristics of M2BPGi}

Serum M2BPGi level increased with subject age (P for trend $<0.001$ ) (Figure 2C). Age, BMI, blood pressure, triglycerides, fasting blood glucose, HbAlc level and were higher in the abnormal M2BPGi group (>1.0) compared with the normal group, and the results were positively correlated with M2BPGi (Table 1). Interestingly, the abnormal M2BPGi group had higher total fat mass and lower ASM. The prevalence of low muscle mass (4.7\%, 48/1,027 vs. $17.4 \%, 8 / 46 ; \mathrm{P}=0.002)$, metabolic syndrome $(14.2 \%, 146 / 1,027$ vs. $30.4 \%, 14 / 46 ; \mathrm{P}=0.003)$, and hypertension $(21.8 \%, 224 / 1,027$ vs. $58.7 \%, 27 / 46 ; \mathrm{P}<0.001)$ were significantly higher in the abnormal M2BPGi group than in the control group (Table 2).

\section{Correlation with hepatic steatosis and hepatic fibrosis burden}

Serum M2BPGi level was higher in NAFLD cases than in the control group (Figure 3A). M2BPGi increased significantly as severity of hepatic steatosis and grade of the fibrosis formulae increased (all P for trend $<0.001$ ). Pearson correlation analysis between M2BPGi and FIB-4 ( $\mathrm{R}=0.293$, $\mathrm{P}<0.001)$, NFS $(\mathrm{R}=0.248, \mathrm{P}<0.001)$ and APRI $(\mathrm{R}=0.238$, $\mathrm{P}<0.001)$ showed a positive relationship. The prevalence of abnormal M2BPGi (>1.0 COI) was higher in a high cutoff for estimated fibrosis formulae (FIB-4, NFS and APRI) compared to low or intermediated cut-offs for estimated fibrosis formulae (Figure 3B,C,D). Prevalence of abnormal M2BPGi in subjects with FIB-4 grade 0 was $3 \%$, and that with FIB-4 grade 2 was $52.9 \%$.

\section{Clinical implications of M2BPGi in diabetes subjects}

The Pearson coefficients between M2BPGi and 
Table 1 Baseline and serological characteristics of study subjects according to M2BPGi level and correlations between M2BPGi level and other variables

\begin{tabular}{|c|c|c|c|c|c|c|}
\hline \multirow{2}{*}{$\begin{array}{l}\text { Health check-up } \\
\text { participants }\end{array}$} & \multicolumn{4}{|c|}{ M2BPGi level (cut off =1.0) } & \multicolumn{2}{|c|}{$\begin{array}{l}\text { Correlations between } \\
\text { M2BPGi and other variables }\end{array}$} \\
\hline & Total $(n=1,073)$ & $\begin{array}{c}\text { Normal, } \\
\mathrm{n}=1,027(95.71)\end{array}$ & $\begin{array}{c}\text { High, } \\
\mathrm{n}=46 \text { (4.29) }\end{array}$ & $\begin{array}{c}\text { P value } \\
\text { (between } \mathrm{N} / \mathrm{H} \text { ) }\end{array}$ & $\begin{array}{l}\text { Pearson } \\
\text { coefficient }\end{array}$ & $P$ value \\
\hline Age (year) & $47.11(11.98)$ & $45.56(11.53)$ & $59.24(15.251)$ & $<0.001$ & 0.323 & $<0.001$ \\
\hline Sex (male) & $626(58.3)$ & $602(58.6)$ & $24(52.2)$ & 0.386 & - & - \\
\hline Minimal activity & 353 (32.9) & $322(31.4)$ & $31(67.4)$ & $<0.001$ & - & - \\
\hline Moderate activity & $431(40.2)$ & $423(41.2)$ & $8(17.4)$ & & & \\
\hline (IPAQ) HEPA & $289(26.9)$ & $282(27.5)$ & $7(15.2)$ & & & \\
\hline \multicolumn{7}{|l|}{ Smoking } \\
\hline Height (cm) & $166.95(8.57)$ & $167.11(8.50)$ & $163.37(9.35)$ & 0.008 & -0.169 & $<0.001$ \\
\hline Body weight (kg) & $65.94(12.61)$ & $65.90(12.47)$ & $66.89(15.45)$ & 0.809 & 0.079 & 0.01 \\
\hline $\mathrm{BMI}\left(\mathrm{kg} / \mathrm{m}^{2}\right)$ & $23.50(3.37)$ & $23.44(3.34)$ & $24.83(3.87)$ & 0.024 & 0.23 & $<0.001$ \\
\hline WC (cm) & $78.56(9.47)$ & $78.35(9.40)$ & $83.26(10.06)$ & 0.001 & 0.227 & $<0.001$ \\
\hline ASM (kg) & $20.39(4.85)$ & $20.44(4.83)$ & $19.33(5.31)$ & 0.136 & -0.069 & 0.024 \\
\hline hSMI (ASM/Ht²) & $7.21(1.13)$ & $7.22(1.13)$ & $7.10(1.22)$ & 0.55 & -0.003 & 0.927 \\
\hline wSMI (ASM/BW ×100\%) & $30.81(3.74)$ & $30.90(3.70)$ & $28.79(3.93)$ & 0.001 & -0.261 & $<0.001$ \\
\hline Platelet count $\left(\times 10^{3} / \mu \mathrm{L}\right)$ & $244.26(51.34)$ & $244.74(50.46)$ & $233.52(67.88)$ & 0.237 & 0.009 & 0.773 \\
\hline Fasting glucose (mg/dL) & $97.35(15.53)$ & $97.04(15.31)$ & $104.21(18.70)$ & 0.001 & 0.212 & $<0.001$ \\
\hline $\mathrm{HbA1c}$ & $5.39(0.56)$ & $5.38(0.56)$ & $5.67(0.66)$ & 0.001 & 0.185 & $<0.001$ \\
\hline Triglycerides (mg/dL) & $120.27(86.13)$ & $117.98(78.87)$ & $171.36(179.27)$ & 0.014 & 0.244 & $<0.001$ \\
\hline Total cholesterol (mg/dL) & $195.72(35.36)$ & $195.50(34.83)$ & $200.58(45.82)$ & 0.391 & 0.086 & 0.005 \\
\hline LDL Cholesterol (mg/dL) & $118.81(26.32)$ & 117.73 (25.99) & $119.71(33.07)$ & 0.764 & 0.113 & $<0.001$ \\
\hline HDL Cholesterol (mg/dL) & $56.17(12.34)$ & $56.22(12.20)$ & $55.19(15.27)$ & 0.386 & -0.149 & $<0.001$ \\
\hline AST (IU/L) & $26.39(12.19)$ & $26.10(11.45)$ & $32.93(22.51)$ & 0.042 & 0.217 & $<0.001$ \\
\hline ALT (IU/L) & $25.09(19.05)$ & $24.86(18.78)$ & $30.23(23.94)$ & 0.122 & 0.182 & $<0.001$ \\
\hline GGT (IU/L) & $26.23(26.26)$ & 25.67 (24.53) & 38.71 (50.34) & 0.106 & 0.208 & $<0.001$ \\
\hline ALP (IU/L) & $67.48(18.79)$ & 67.05 (18.43) & 77.06 (23.82) & 0.003 & 0.265 & $<0.001$ \\
\hline
\end{tabular}

Table 1 (continued) 
Table 1 (continued)

\begin{tabular}{|c|c|c|c|c|c|c|}
\hline \multirow{2}{*}{$\begin{array}{l}\text { Health check-up } \\
\text { participants }\end{array}$} & \multicolumn{4}{|c|}{ M2BPGi level (cut off =1.0) } & \multicolumn{2}{|c|}{$\begin{array}{l}\text { Correlations between } \\
\text { M2BPGi and other variables }\end{array}$} \\
\hline & Total $(n=1,073)$ & $\begin{array}{c}\text { Normal, } \\
\mathrm{n}=1,027(95.71)\end{array}$ & $\begin{array}{c}\text { High, } \\
\mathrm{n}=46(4.29)\end{array}$ & $\begin{array}{c}\text { P value } \\
\text { (between } \mathrm{N} / \mathrm{H} \text { ) }\end{array}$ & $\begin{array}{l}\text { Pearson } \\
\text { coefficient }\end{array}$ & $P$ value \\
\hline Total Bil (IU/L) & $0.9015(0.3587)$ & $0.9878(0.3595)$ & $0.7620(0.3128)$ & 0.002 & -0.44 & 0.146 \\
\hline Direct Bil (IU/L) & $0.1664(0.0616)$ & $0.1675(0.0617)$ & $0.1428(0.0555)$ & 0.007 & -0.073 & 0.016 \\
\hline NAFLD & $602(56.1)$ & $570(55.5)$ & $32(69.6)$ & 0.06 & - & - \\
\hline Fatty liver index & $22.55(22.58)$ & $22.01(22.13)$ & $24.39(28.83)$ & 0.001 & 0.257 & $<0.001$ \\
\hline AST to ALT ratio & $1.2353(0.4230)$ & $1.2331(0.4158)$ & $1.2839(0.5627)$ & 0.426 & -0.095 & 0.002 \\
\hline AST to Platelet ratio index & $0.2843(0.1553)$ & $0.2791(0.1367)$ & $0.3992(0.3665)$ & $<0.001$ & 0.238 & $<0.001$ \\
\hline M2BPGi (COI) & $0.5116(0.2642)$ & $0.4744(0.1849)$ & $1.3410(0.3862)$ & $<0.001$ & 1 & $<0.001$ \\
\hline
\end{tabular}

Data are presented as mean (SD) or number (percent). Mann-Whitney $U$ test was used to compare continuous variables between groups. HEPA, health-enhancing physical activity; BMI, body weight index; WC, waist circumference; ASM, appendicular skeletal muscle; w/h SMI, weight or height adjusted skeletal muscle index; SBP/DBP, systolic/diastolic blood pressure; LDL/HDL, low/high density lipoprotein; AST, aspartate aminotransferase; ALT, alanine aminotransferase; GGT, gamma glutamyl transferase; ALP, alkaline phosphatase; Bil, bilirubin; NAFLD, non-alcoholic fatty liver disease; FIB-4, fibrosis-4 index; NFS, NAFLD fibrosis score; M2BPGi, Mac-2 binding protein glycosylation isomer.

estimated fibrosis values (FIB-4, NFS and APRI) were higher in diabetes group than in the general population (Figure $4 A, B, C$ ). However, Pearson coefficients between M2BPGi and estimated hepatic fibrosis burden were similar to those in the NAFLD and MetS groups.

The prevalence of advanced fibrosis $(>2.67 \mathrm{FIB}-4)$ in cases with abnormal M2BPGi ( $>1.0$ COI) was $19.6 \%$ in the general population (Figure $4 D$ ) and $50 \%$ in diabetes. The sensitivity and specificity of abnormal M2BPGi (>1.0 COI) in diabetes patients for predicting advanced fibrosis were $50 \%$ and $95.6 \%$, respectively.

\section{Discussion}

This data showed that the reference value of M2BPGi was 0.5-1.0 COI in the average risk group. Serum M2BPGi value was positively correlated with metabolic parameters as well as age. While the prevalence of advanced fibrosis in the total population was just $1.6 \%$ (FIB-4 $>2.65$ ), the prevalence of advanced fibrosis increased to $50 \%$ in the high M2BPGi group with diabetes. There was a high possibility of advanced hepatic fibrosis in subjects with abnormal M2BPGi level (>1.0) in diabetes.
These results are consistent with previous studies such as Kang et al., which reported conditions in health check-up settings ( $1.3 \%$ in the average risk group, $1.5 \%$ in NAFLD, $4.3 \%$ in T2DM) and Loomba et al.'s reports in diabetes (7.1\%) that used MRE for diagnosing advanced liver fibrosis $(\geq \mathrm{F} 3)(2,18)$. Therefore, the M2BPGi test is useful for advanced liver fibrosis screening, even if there are no risk factors for other liver diseases in subjects with impaired fasting glucose or diabetes.

To the best of our knowledge, this is the first study to compare the hepatic fibrosis burden according to underlying conditions, such as NAFLD and diabetes and M2BPGi abnormality in an average risk group. Kamada et al. also conducted a study in an average risk group, but their study focused only on predicting the proportion of suspected advanced hepatic fibrosis patients in a healthy cohort by a cut-off value based on a biopsy-proven cohort. However, our study focused on clinical usage of M2BPGi in an average risk group and further analysed the hepatic fibrosis burden of subjects according to underlying condition and M2BPGi abnormality. As a result, because we know that patients with diabetes identified in the high M2BPGi group in a health check-up have a relatively high fibrosis burden, 

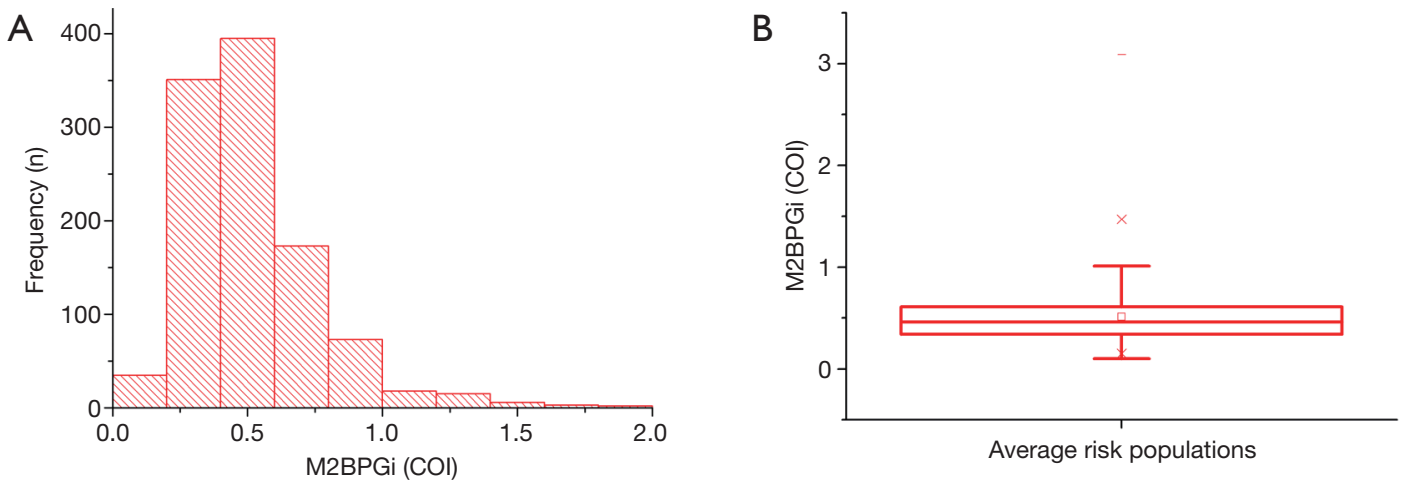

Descriptive statistics

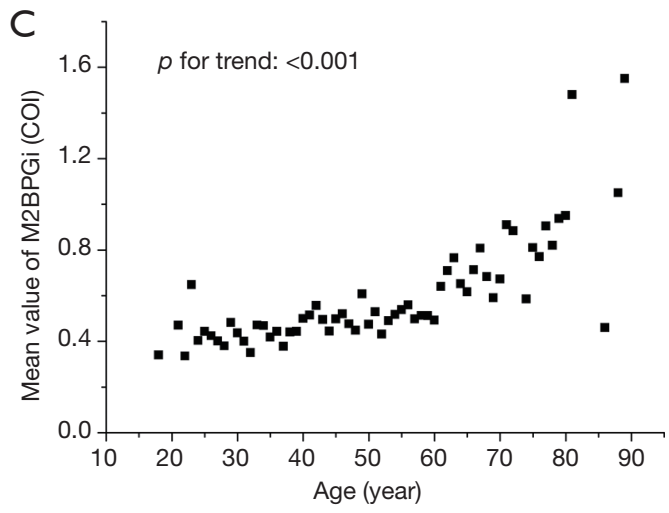

Figure 2 Histogram (A) and Box plot (B) of M2BPGi in average risk population ( $\mathrm{n}=1,073)$. (C) The graph of M2BPGi level of study subjects according to age. M2BPGi, Mac-2 binding protein glycosylation isomer; Hi, high.

Table 2 Odds ratios (95\% CIs) of main categorical variables for high M2BPGi

\begin{tabular}{|c|c|c|c|c|}
\hline \multirow{2}{*}{ Health check-up, $n=1,073(M=626 / F=447)$} & \multicolumn{2}{|c|}{ M2BPGi (cut off =1.00) } & \multirow{2}{*}{ Odds ratio $(95 \% \mathrm{Cl})$} & \multirow{2}{*}{$P$ value } \\
\hline & Low, $n=1,027$ & High, $n=46$ & & \\
\hline Age $\geq 60(14.4)$ & $131(12.8)$ & $24(52.2)$ & $7.461(4.067-13.689)$ & $<0.001$ \\
\hline $\mathrm{BMI} \geq 25$ (29.5) & 297 (28.9) & $19(41.3)$ & $1.730(0.947-3.159)$ & 0.071 \\
\hline Low muscle mass by hSMI (1.2) & $9(0.9)$ & $4(8.7)$ & $10.772(3.188-36.399)$ & 0.002 \\
\hline MetS (14.9) & $146(14.2)$ & $14(30.4)$ & $2.640(1.375-5.067)$ & 0.003 \\
\hline High BP (34.9) & $343(33.4)$ & $31(67.4)$ & $4.121(2.195-7.738)$ & $<0.001$ \\
\hline Abdominal obesity (13.5) & $131(12.8)$ & $14(30.4)$ & $2.992(1.556-5.756)$ & 0.001 \\
\hline IFG (32.0) & $320(31.2)$ & $23(50.0)$ & $2.209(1.221-3.997)$ & 0.007 \\
\hline T2DM (6.9) & $68(6.6)$ & $6(13.0)$ & $2.115(0.866-5.165)$ & 0.125 \\
\hline NAFLD (56.1) & $570(55.5)$ & $32(69.6)$ & $1.833(0.966-3.475)$ & 0.06 \\
\hline
\end{tabular}

Data are presented as number (percent). Chi-square test or Fisher's Exact test was used to compare groups. Fisher's exact test was used in the presence of a cell expected to have a count less than five. BMI, body weight index; w/h SMI, weight or height adjusted skeletal muscle index; MetS, metabolic syndrome; IFG, impaired fasting glucose; TG, triglycerides; HDL, high density lipoprotein; HTN, hypertension; T2DM, type-2 diabetes mellitus; NAFLD, non-alcoholic fatty liver disease. 

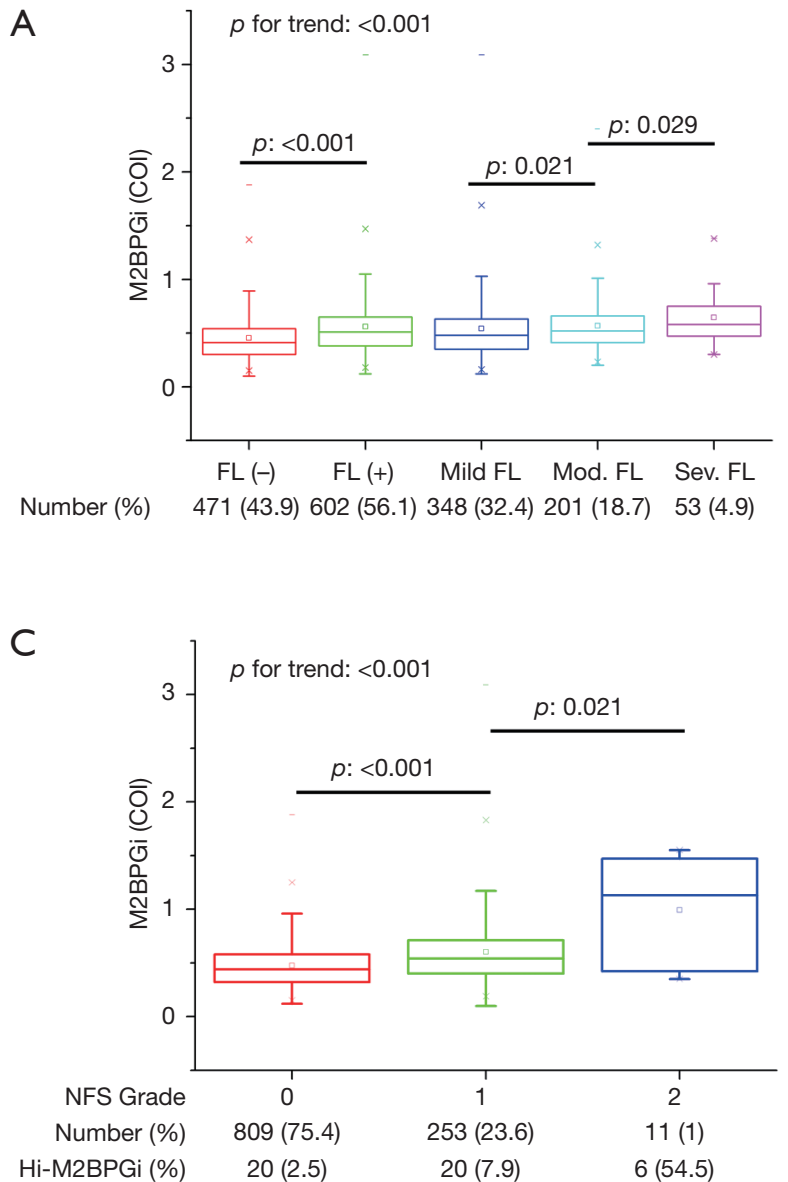
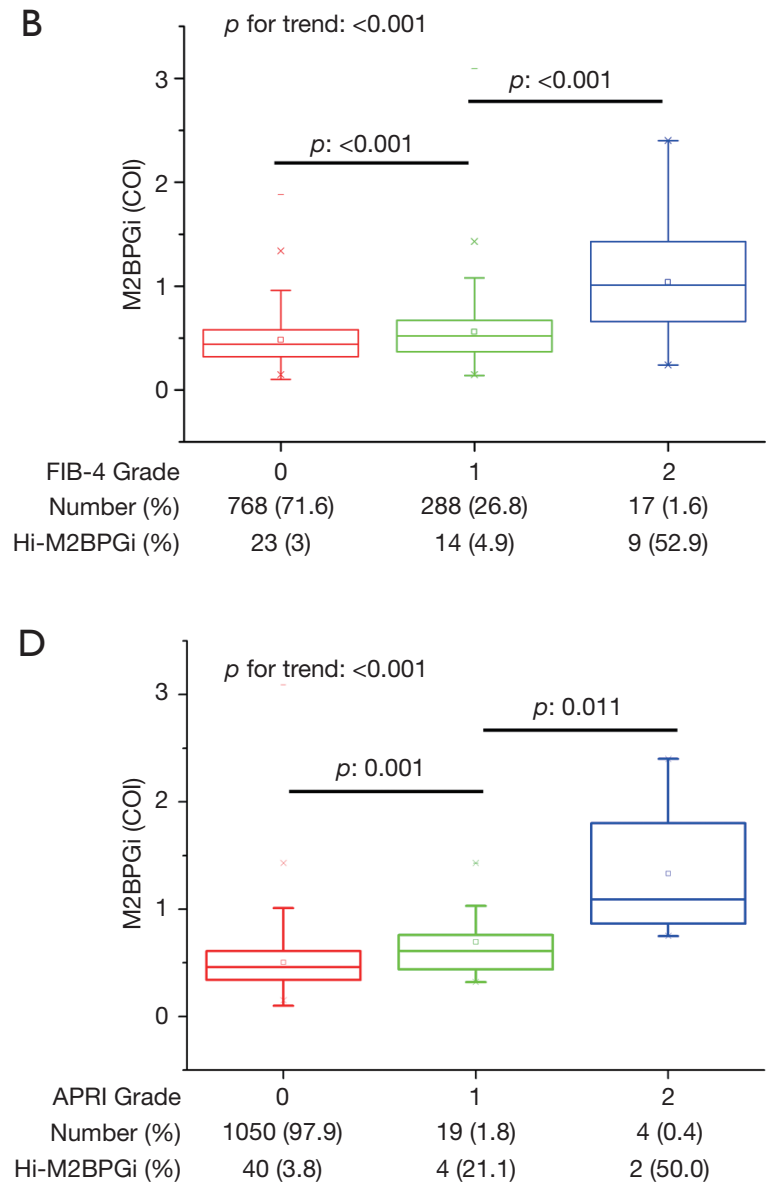

Figure 3 Box plot of M2BPGi versus hepatic steatosis status (A), FIB-4 (B), NFS (C), and APRI grade (D). Kruskale Wallis tests. was used for comparing each group. Fibrosis scoring systems such as FIB-4, NFS, and APRI are graded on a scale 0, 1, 2. M2BPGi, Mac-2 binding protein glycosylation isomer; Hi, high; FL, fatty liver; Mod, moderate; Sev, severe; FIB-4, fibrosis-4 index; NFS, NAFLD fibrosis score; APRI, AST to platelet ratio index.

we recommend that they be evaluated immediately.

In the European NAFLD guidelines, screening for insulin resistance, metabolic syndrome, or T2DM was emphasized for NAFLD patients (19). Many previous reports have indicated that diabetes is strongly associated with NASH, including mild and advanced fibrosis and lipotoxins, mitochondrial function, cytokines, and adipocytokines have been proposed to play a major part in both NAFLD and T2DM (20,21). Moreover, several studies have compared the prevalence of advanced liver fibrosis in patients with NAFLD and/or diabetes using transient elastography in primary care or in health check-up settings. In their studies, the prevalence of advanced liver fibrosis in NAFLD patients with T2DM was much higher than in the average risk group, though the prevalence was less than $20 \%$ overall $(2,18,22,23)$. The prevalence of advanced fibrosis based on FIB-4 was highest in the abnormal M2BPGi group with diabetes and increased from $4.4 \%$ to $50 \%$ (11.36 times) in the abnormal M2BPGi group with diabetes compared to the normal M2BPGi group with diabetes, and the odds ratio was 21.667 (CI: 3.007-156.141, $\mathrm{P}=0.006$ ). Similar result was also observed, when other fibrosis scoring formulae, such as NFS and APRI was used (Figure S1). These results indicate that M2BPGi is representative of hepatic fibrosis, and that subjects in the abnormal M2BPGi category with diabetes should be suspected to have a higher fibrosis burden than the control group. Moreover, the low cost and simple procedure involved with using a single blood sample allow M2BPGi analysis to be applied for screening advanced hepatic fibrosis; in particular, this approach is more suitable 
Correlation between M2BPGi and liver fibrosis formulae

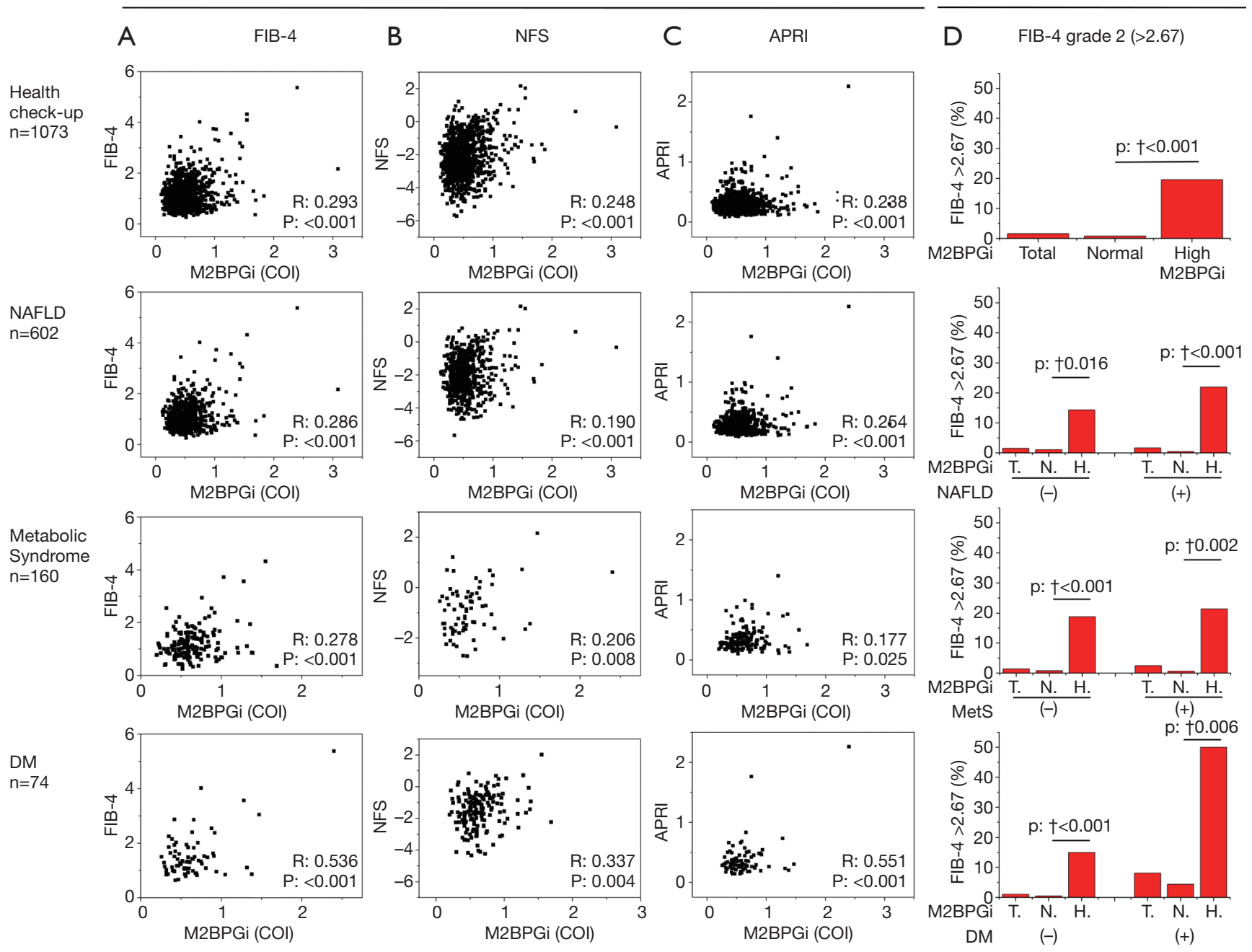

Figure 4 The scatter plot of M2BPGi level versus (A) FIB-4, (B) NFS (C) APRI for health check-up, NAFLD, metabolic syndrome or DM groups. Pearson correlation coefficients and p-values are presented in the graph. The prevalence of advanced fibrosis (D) in subjects with and without NAFLD, MetS or DM. Normal group $(\mathrm{N})$ and High M2BPGi group $(\mathrm{H})$ were divided at the cut-off value of 1.0. T indicate total subjects. Chi-square test or Fisher's Exact test was used for categorical variables to compare between groups. M2BPGi, Mac-2 binding protein glycosylation isomer; NAFLD, non-alcoholic fatty liver disease; FIB-4, fibrosis-4 index; NFS, NAFLD fibrosis score; APRI, AST to platelet ratio index; MetS, metabolic syndrome; DM, diabetes mellitus.

for primary clinical or health check-up settings.

This study has several limitations. First, estimated fibrosis values of FIB-4 and NFS were used to diagnose advanced fibrosis. Gold standard diagnostic tools such as liver biopsy or MRE would have been better for diagnosis for advanced fibrosis. Second, there were fewer abnormal M2BPGi subjects in the entire subject group. Therefore, large-scale studies will be needed for a more accurate analysis. Third, further study is needed to compare the performance of M2BPGi with those of FIB-4 and NFS, which are frequently used for screening of liver fibrosis, or merged methods.

In conclusion, M2BPGi could be used to differentiate high-risk patients with advanced fibrosis from the general population using a cut-off of 1.0 (>2SD). Moreover, when the abnormal M2BPGi group had a diagnosis of diabetes, the prevalence of advanced fibrosis increased to $50 \%$. Therefore, patients in the high M2BPGi group with diabetes in a health check-up have a relatively high fibrosis burden and should undergo further evaluation. 


\section{Acknowledgments}

Funding: This study was supported by the Sysmex Company. The funding source had no role in the study design, implementation, data collection, analysis, interpretation, or in the preparation, review, or approval of the manuscript.

\section{Footnote}

Reporting Checklist: The authors have completed the STROBE reporting checklist. Available at http://dx.doi. org/10.21037/atm-20-5216

Data Sharing Statement: Available at http://dx.doi. org/10.21037/atm-20-5216

Conflicts of Interest: All authors have completed the ICMJE uniform disclosure form (available at http://dx.doi. org/10.21037/atm-20-5216). All authors have no competing or conflicts of interest to declare.

Ethical Statement: The authors are accountable for all aspects of the work in ensuring that questions related to the accuracy or integrity of any part of the work are appropriately investigated and resolved. The study was conducted in accordance with the Declaration of Helsinki (as revised in 2013). The Institutional Review Board (IRB) of Hanyang University Medical Centre approved this study protocol (IRB No. 2018-12-020). The protocol was also registered at the Clinical Research Information Service (https://cris.nih.go.kr/cris, Registration No. KCT0004462). The informed consent was waived, because suspected harm to participants was minimal or none.

Open Access Statement: This is an Open Access article distributed in accordance with the Creative Commons Attribution-NonCommercial-NoDerivs 4.0 International License (CC BY-NC-ND 4.0), which permits the noncommercial replication and distribution of the article with the strict proviso that no changes or edits are made and the original work is properly cited (including links to both the formal publication through the relevant DOI and the license). See: https://creativecommons.org/licenses/by-nc-nd/4.0/.

\section{References}

1. Jeong EH, Jun DW, Cho YK, et al. Regional prevalence of non-alcoholic fatty liver disease in Seoul and Gyeonggi- do, Korea. Clin Mol Hepatol 2013;19:266-72.

2. Kang KA, Jun DW, Kim MS, et al. Prevalence of significant hepatic fibrosis using magnetic resonance elastography in a health check-up clinic population. Aliment Pharmacol Ther 2020;51:388-96.

3. Musso G, Gambino R, Cassader M, et al. Meta-analysis: natural history of non-alcoholic fatty liver disease (NAFLD) and diagnostic accuracy of non-invasive tests for liver disease severity. Ann Med 2011;43:617-49.

4. Chen J, Talwalkar JA, Yin M, et al. Early detection of nonalcoholic steatohepatitis in patients with nonalcoholic fatty liver disease by using MR elastography. Radiology 2011;259:749-56.

5. Wai CT, Greenson JK, Fontana RJ, et al. A simple noninvasive index can predict both significant fibrosis and cirrhosis in patients with chronic hepatitis C. Hepatology 2003;38:518-26.

6. Vallet-Pichard A, Mallet V, Nalpas B, et al. FIB-4: an inexpensive and accurate marker of fibrosis in $\mathrm{HCV}$ infection. comparison with liver biopsy and fibrotest. Hepatology 2007;46:32-6.

7. Yu SJ, Kim DH, Lee JH, et al. Validation of P2/MS and other noninvasive fibrosis scoring systems in the Korean population with nonalcoholic fatty liver disease. Korean J Gastroenterol 2011;57:19-27.

8. Lim DH, Kim M, Jun DW, et al. Diagnostic Performance of Serum Asialo alpha1-Acid Glycoprotein Levels to Predict Liver Cirrhosis. Gut Liver 2020. [Epub ahead of print].

9. Atsukawa M, Tsubota A, Okubo T, et al. Serum Wisteria floribunda agglutinin-positive Mac-2 binding protein more reliably distinguishes liver fibrosis stages in non-alcoholic fatty liver disease than serum Mac-2 binding protein. Hepatol Res 2018;48:424-32.

10. Cho SY, Hur M. Mac-2 Binding Protein Glycosylation Isomer: Emerging Non-Invasive Serum Marker for Liver Fibrosis. Ann Lab Med 2018;38:289-90.

11. Shirabe K, Bekki Y, Gantumur D, et al. Mac-2 binding protein glycan isomer (M2BPGi) is a new serum biomarker for assessing liver fibrosis: more than a biomarker of liver fibrosis. J Gastroenterol 2018;53:819-26.

12. Koo BK, Kim D, Joo SK, et al. Sarcopenia is an independent risk factor for non-alcoholic steatohepatitis and significant fibrosis. J Hepatol 2017;66:123-31.

13. Bedogni G, Bellentani S, Miglioli L, et al. The Fatty Liver Index: a simple and accurate predictor of hepatic steatosis in the general population. BMC Gastroenterol 2006;6:33.

14. Lin ZH, Xin YN, Dong QJ, et al. Performance of the 
aspartate aminotransferase-to-platelet ratio index for the staging of hepatitis C-related fibrosis: an updated metaanalysis. Hepatology 2011;53:726-36.

15. Grundy SM, Brewer HB Jr, Cleeman JI, et al. Definition of metabolic syndrome: Report of the National Heart, Lung, and Blood Institute/American Heart Association conference on scientific issues related to definition. Circulation 2004;109:433-8.

16. Lee SY, Park HS, Kim DJ, et al. Appropriate waist circumference cutoff points for central obesity in Korean adults. Diabetes Res Clin Pract 2007;75:72-80.

17. Mittelstaedt C. Retroperitoneum. General ultrasound New York: Churchill Livingstone 1992;749.

18. Doycheva I, Cui J, Nguyen P, et al. Non-invasive screening of diabetics in primary care for NAFLD and advanced fibrosis by MRI and MRE. Aliment Pharmacol Ther 2016;43:83-95.

Cite this article as: Park H, Jun DW, Park HK, Kim M. Clinical implications of serum Mac-2-binding protein glycan isomer as a novel biomarker of advanced hepatic fibrosis in diabetes. Ann Transl Med 2020;8(23):1583. doi: 10.21037/atm20-5216
19. EASL-EASD-EASO Clinical Practice Guidelines for the management of non-alcoholic fatty liver disease. J Hepatol 2016;64:1388-402.

20. Loomba R, Abraham M, Unalp A, et al. Association between diabetes, family history of diabetes, and risk of nonalcoholic steatohepatitis and fibrosis. Hepatology 2012;56:943-51.

21. Tilg H, Moschen AR, Roden M. NAFLD and diabetes mellitus. Nat Rev Gastroenterol Hepatol 2017;14:32-42.

22. Koehler EM, Plompen EP, Schouten JN, et al. Presence of diabetes mellitus and steatosis is associated with liver stiffness in a general population: The Rotterdam study. Hepatology 2016;63:138-47.

23. Kwok R, Choi KC, Wong GL, et al. Screening diabetic patients for non-alcoholic fatty liver disease with controlled attenuation parameter and liver stiffness measurements: a prospective cohort study. Gut 2016;65:1359-68. 


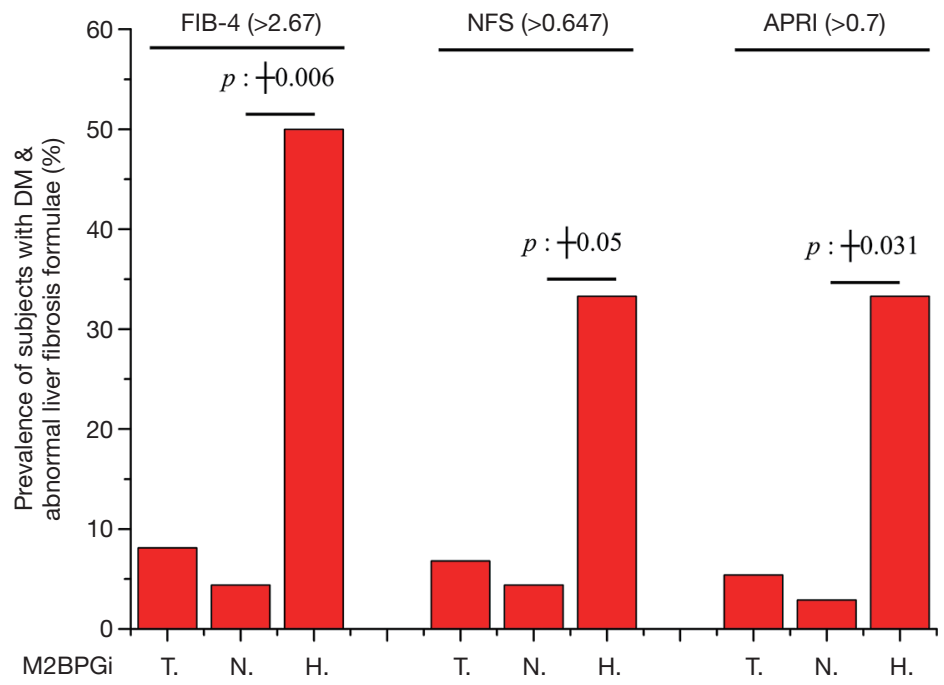

Figure S1 The prevalence of diabetes subjects with abnormality of liver fibrosis formulae such as FIB-4, NFS or APRI according to abnormality of M2BPGi. Normal group $(\mathrm{N})$ and High M2BPGi group $(\mathrm{H})$ were divided at the cut-off value of 1.0. T indicate total subjects. Chi-square test or Fisher's Exact test was used for categorical variables to compare between groups. Normal group (N) and High M2BPGi group $(\mathrm{H})$ were divided at the cut-off value of 1.0. T indicate total subjects. Chi-square test or Fisher's Exact test was used for categorical variables to compare between groups. M2BPGi, Mac-2 binding protein glycosylation isomer; NAFLD, non-alcoholic fatty liver disease; FIB-4, fibrosis-4 index; NFS, NAFLD fibrosis score; APRI, AST to platelet ratio index; DM, diabetes mellitus. 